\title{
ANALYSING AND SOLVING THE REDUCED-ABILITY AND EXCESSIVE-USE DILEMMAS IN TECHNOLOGY USE
}

\author{
Wesugi, Shigeru \\ Waseda University
}

\begin{abstract}
Technology use provides great benefits by extending human ability, but the negative effects cannot always be ignored. The author examined the dilemmas of technology use based on Shibata's analysis of McLuhan's extension theory and indicated two types of dilemmas in continuous technology use. First is the decreased human ability as the innate functionality is substituted by the technology. Second is excessive utilisation of the technology, which may instil a false sense of naturally extended ability.

Subsequently, the author considered and suggested approaches for mitigating both types of dilemmas. The decreased-ability dilemma might be resolved by continuously utilising the technology and designing technology relevant to the degree of human ability. Meanwhile, the excessive-utilisation dilemma might be resolved by regulating the technology use and designing technology that achieves the desired disposition change in users.

Finally, the possibility of advancing the existing design approaches to further resolve the dilemmas was discussed.
\end{abstract}

Keywords: Design method, Early design phases, Ethics, Technology, User centred design

Contact:

Wesugi, Shigeru

Waseda University

Faculty of Science and Engineering

Japan

wesugi@waseda.jp

Cite this article: Wesugi, S. (2019) 'Analysing and Solving the Reduced-Ability and Excessive-Use Dilemmas in Technology Use', in Proceedings of the 22nd International Conference on Engineering Design (ICED19), Delft, The Netherlands, 5-8 August 2019. DOI:10.1017/dsi.2019.145 


\section{INTRODUCTION}

Technologies are an essential component of modern human lives. At the start of the day, humans are woken by a smartphone that presents the schedule for that day. A microwave oven then heats their breakfasts and a washing machine cleans their dishes. A motorised vehicle transports them to their working places. Modern lifestyles depend on numerous technologies that connect both directly and indirectly with users. Some technologies seemingly provide only benefits, but other technologies impact negatively as well as positively, posing a dilemma in technology use.

For example, people using smartphones while walking can accidentally collide with other walkers. Such users are referred to as smartphone zombies because they pay less attention to their surroundings than to the contents of their smartphones. To mitigate this social problem, announcements and public posters encourage users to self-regulate their smartphone use on crowded walkways and platforms. However, the numbers of accidents caused by walking smartphone users remain high.

The dual positive and negative effects of technology have been recognised for some time. Table 1 lists some familiar dilemmas posed by these effects (Tsuchiya, 2001). New dilemmas seem to accompany each deployment of a novel technology.

Table 1. Technology as a double-edged sword

\begin{tabular}{|l|l|l|l|}
\hline \multicolumn{1}{|c|}{ Technology } & \multicolumn{1}{|c|}{ Negative effect } & \multicolumn{1}{c|}{ Technology } & \multicolumn{1}{c|}{ Negative effect } \\
\hline Calculator & $\begin{array}{l}\text { Less mental calculation } \\
\text { ability }\end{array}$ & Instant food & Less finger movement \\
\hline Autofocus camera & $\begin{array}{l}\text { Less visual measurement } \\
\text { ability }\end{array}$ & Disposal diaper & Less sensitivity of baby \\
\hline Air conditioner & Inadequate exercise & Baby basket & Less physical contact \\
\hline Intercom & Inadequate exercise & Facsimile & Increasing sheets of paper \\
\hline Comfortable chair & Drowsy & Bullet train & Increasing hours of labour \\
\hline
\end{tabular}

*This table was partly excerpted and translated by the author.

After serious social impacts, most of these dilemmas are being addressed by supportive measures, which vary from issuing a warning to regulating by rules. Due to the multistability of technology (Ihde 1990, Verbeek 2011), we cannot predict all possible consequences of technology use. Consequently, the context of technology usage can differ from the designed context. For example, global positioning systems have evolved from military technologies to navigation devices for driving and hiking.

When a technology use becomes habitual and its negative effects persist, the problems become more difficult to resolve. Such intractable problems can be addressed through a variety of approaches, such as establishing legal and literacy education systems for technology.

In this paper, the author analyses and characterises several dilemmas in technology use. To mitigate such dilemmas, the author then proposes original schemes and repositions some existing methodologies. Finally, the author discusses the possibility of advancing the existing design approaches to resolve these dilemmas.

\section{RELATIONS BETWEEN HUMAN BEINGS AND TECHNOLOGIES}

\subsection{Dichotomy between subject and object}

Before analysing the dilemmas in technology use, the author examines the fundamental relations between humans and technologies, how humans experience those technologies, and how the technologies present to humans. The technologies mentioned in this paper are material and directly contact their human users. They include (but are not limited to) tools, devices, apparatuses, and machines. However, a language or a legal system regulating their use in society is lacking for these technologies. In contrast, medical technologies are already regulated by the legal system, so are excluded from the present study.

Conceptually, humans and technologies are often regarded as subjects and objects, respectively. Humans (or subjects) are the agents that voluntarily manipulate the technology (or object). The object is merely a physical entity that differs from humans. Challenging the traditional dichotomy of subjects and objects, Verbeek (2015) divided the relations between humans and technologies into three viewspoints: extension, dialectics and hybrids. 
The 'extension' and 'dialectics' views comprise a dichotomy. 'Extension' literally means that the technology enhances human functionality and is incorporated into humans. The humans are responsible for using the technology, whereas the technology is neutral.

In contrast, the 'dialectics' view highlights the opposing nature of humans and technologies, and the strain in the human-technology relationship. From one perspective, dialectics asks how humans can be freed from the suppression of technology. From another perspective, it argues that technology is externalized human function; however, it is approaching the capacity to perform human functionalities.

The 'hybrid' view posits a reciprocal relation beyond the human-technology dichotomy. Human beings create technologies while technologies shape human beings. Accordingly, technologies alter how human beings perceive and act. This symbiotic relationship between humans and technologies leads to methodologies that aid the development of a better life, and is consistent with ancient Greek ethics and tool philosophy in Japanese culture.

Recently, these complementary relations have been actively explored in post-phenomenology. Working from a phenomenological viewpoint, Ihde identified four basic relations between humans and technologies: embodiment, hermeneutics, alterity, and background (Ihde, 1990, Verbeek, 2011). In an embodiment relation, the technology becomes part of the human body (e.g. eye glasses). In a hermeneutic relation, the technology represents a world phenomenon to be interpreted by humans (e.g. a thermometer). In an alterity relation, the technology interacts humans as other beings (e.g. a vending machine). In a background relation, the technology works automatically in the background (e.g. a refrigerator).

\subsection{Three types of extension}

In the history of thought, Shibata examined McLuhan's concept of technological devices as an extension of the body, and categorized three types of extensions based on their origins as given in literature reports (Shibata, 2013).

In the first type of extension, 'the natural bodily functions extend by substituting artefacts for natural organs'. Shibata indicated that this type of extension includes substitute, replace, enhance, augment, and/or amplify. This type of extension was followed by Vannevar Bush (computer), Douglas Engelbart (mouse), Simon Ramo (ICBM), Kevin Warwick (cyborg), and others, and originated in Plato's 'Phaedrus' (Plato) and 'Zhuang-zi' (Zhuang-zi). The classical literature also mentioned the dilemmas in utilising the technologies. In the next section, these dilemmas will be discussed in detail.

The alterity and background relations explained in 2.1 also fall into this type of extension, because such technology can be thought to substitute human organs. Moreover, this type of extension often incorporates novel technology. The author refers to this type of extension as the 'substituting type'.

The second extension posits that 'the boundary between the tool and the body extends and the tool becomes a part of the body'. As explained by Shibata, this type of extension includes boundary and prolonging. For instance, a white cane that has been mastered by a visually impaired person becomes a part of that person's body (citing Maurice Merleau-Ponty and Michael Polanyi). The earliest literary reference to this extension type was the dualism concept of René Descartes, which considers the body as separate from other things. Therefore, this type of extension is called the 'prolonging type' in this paper.

The embodiment relations explained in 2.1 are precisely located in this type. The hermeneutics relations can also be included here, because they indicate how humans interpret the border between the body and all other entities.

The third extension posits that 'an artefact is the projection of human organ. And it is highlighted that through externalisation, the hidden mechanism is discovered.' This type of extension includes externalisation and outering (citing Ernst Kapp and André Leroi-Gourhan), and can be considered to originate from the medical technique of Hippocrates (namely, that the contents discharged from the body diagnostically indicate the body's internal condition). Therefore, the author refers to this extension as the 'externalizing type'. The dialectics relations explained in 2.1 belong to this type of extension.

Separating humans and technologies is inadequate for analysing the dilemmas in technology usage, because technology use itself has altered how humans perceive, behave with and relate to the technologies. Therefore, the author adopts the 'hybrid' view and examines the dilemmas using extension theory in the next section. 


\section{ANALYZING DILEMMAS IN TECHNOLOGY USE}

\subsection{Literary-based origin of technological dilemmas}

The author focusses on the analysis of Shibata, who examined the dilemma described in Phaedrus (Plato). Shibata's analysis seems to be a basis for examining other dilemmas. In Phaedrus, the inventor Theuth invented letters that would improve the wisdom and memory power of users, and introduced them to the Egyptian king Thamus. However, Thamus declared that the letters would create forgetfulness because users would swap memorising for writing, and complacency because users would gain knowledge of the technology without appreciating the source of their improved ability. Hence, the introduction of this technology created a dichotomy between improving memory ability and creating forgetfulness. The other negative effect, complacency, creates another dichotomy between being knowledgeable and developing a know-it-all attitude.

According to Shibata's analysis, the memory improvements touted by Theuth presume that letters are available anytime, whereas the forgetfulness concerns of Thamus are sourced from unavailability of letters (Shibata, 2015). That is, whether the letters foster memory building or cause forgetfulness depends on the continuity of the technology use.

To gain insight into the dilemmas of technology usage, the author revisits two types of dilemmas described in Phaedrus and Zhuang-zi, and reconsiders them in terms of 'continuity of technology use'.

\subsection{Decreased human ability after introducing substituting-type extension technology}

The first dilemma is whether to withhold the technology or risk creating forgetfulness (which decreases human ability). For example, a calculator reduces the user's mental calculation ability and a word processor creates forgetfulness of word spellings. When the use of technology is discontinued, the decreased ability becomes apparent. Because the technology substitutes a human function, the natural ability reduces. In this paper, such a dilemma is referred to as the 'decreased ability type'. The characteristics of this dilemma will be demonstrated in the following schema. Human abilities to complete a specific function can be represented as a set of ability members $A_{1}, A_{2}, \ldots, A_{n}$, where a higher index indicates a higher level of ability.

- $\quad$ Natural human ability before technology use $=\left\{A_{1}, A_{2}, \ldots, A_{n}\right\}$

- Human ability while using technology $=\left\{A_{1}, A_{2}, \ldots, A_{n-i}\right\}$

- Function gained by using the technology $=\left\{A_{n-i+1}, A_{n-i+2}, \ldots, A_{n}, \ldots, A_{n+j}\right\}$

- Integration of human ability and technology use $=\left\{A_{1}, A_{2}, \ldots, A_{n-i}\right\}+\left\{A_{n-i+1}, A_{n-i+2}, \ldots, A_{n}, \ldots, A_{n+j}\right\}$

Before utilising the technology, a user's natural ability is $\left\{A_{1}, A_{2}, \ldots, A_{n}\right\}$. The technology use substitutes $\left\{A_{n-i+1}, \ldots, A_{n}\right\}$ and additionally enhances $\left\{A_{n+1}, \ldots, A_{n+j}\right\}$. When a user loses the technology, he or she loses not only the enhanced ability $\left\{A_{n+1}, \ldots, A_{n+j}\right\}$, but also the substituted ability $\left\{A_{n-i+1}, \ldots, A_{n}\right\}$. The shortfall, which soon becomes apparent, is $\left\{A_{n-i+1}, \ldots, A_{n}\right\}$. In this schema, an increase and decrease in the number of members indicates an enhancement and degradation of ability, respectively. Thereby, users acquire an image of linearity and reversibility in technology use.

\subsection{Complacency after utilizing prolonging-type extension technology}

The second dilemma is whether to withdraw the technology or risk the knowledgeable but naive use of the technology. The literal origins of this dilemma were 'Phaedrus' and 'Zhuang-zi' (Zhuang-zi) (Shibata, 2013); in this paper, Zhuang-zi is focussed upon. In one episode of this work, an aged man declined to use an effective tool for lifting water because he believed that his preoccupation with the tool use would alter his disposition. This type of dilemma indicates that human dispositions can be altered by long-term (but not by short-term) usage of a technology.

This type of dilemma might fall into the decreased-ability type, because both dilemmas can be interpreted as negative effects on the natural human condition, rousing concern in users.

On the other hand, this type of dilemma might arise when users regard their enhanced ability as a natural advance in their own ability, while paying less attention to the technology itself. In this situation, the technology becomes embodied in the user's body. Therefore, this type of dilemma is considered as a prolonging type of extension technology. Its characteristics are illustrated in the following schema. 
- $\quad$ Natural human ability before technology use $=\left\{A_{1}, A_{2}, \ldots, A_{n}\right\}$

- Human ability while using the technology $=\left\{A_{1}, A_{2}, \ldots, A_{n-i}^{\prime}, \ldots, A_{n-1}^{\prime}, A_{n}^{\prime}\right\}$

- $\quad$ Function gained by the embodied technology $=\left\{A_{n-i}, \ldots, A_{n-1}, A_{n}, \ldots, A_{n+j}\right\}$

- Integration of human ability and technology $=\left\{A_{1}, A_{2}, \ldots, A_{n-i}^{\prime}, \ldots, A_{n-1}^{\prime}, A_{n}^{\prime}\right\}$ $+\left\{A_{n-i}, \ldots, A_{n-1}, A_{n}, \ldots, A_{n+j}\right\}$

Before using the technology, a user's natural ability is $\left\{A_{1}, A_{2}, \ldots, A_{n}\right\}$. When using the technology, the human ability integrates with the technology, and the ability extends to $\left\{A_{1}, A_{2}, \ldots, A_{n-i}^{\prime}, \ldots, A_{n-1}^{\prime}, A_{n}^{\prime}\right\}$ $+\left\{A_{n-i}, \ldots, A_{n-1}, A_{n}, \ldots, A_{n+j}\right\}$. Note that part of the human ability during technology use has altered from $\left\{A_{n-i}, \ldots, A_{n-1}, A_{n}\right\}$ to $\left\{A_{n-i}^{\prime}, \ldots, A_{n-1}^{\prime}, A_{n}^{\prime}\right\}$. This schema shows that though the human ability alters around the human-technology boundary, the users regard the altered ability as their own ability, and will readily disregard the boundary. Therefore, the user pays little attention to the technology use. In this situation, users operate the technology knowledgeably but without a realistic appreciation of their altered ability. This causes the sense of complacency that worried Thamus in Phaedrus.

The convenient e-mail system, which also increases the workload, and an energy-saving lighting apparatus deployed in many spaces, fall into this type of dilemma. Especially, the latter case creates a 'rebound effect' in which the technology that supposedly reduces the energy and material requirements instead increases these requirements. Therefore, dilemmas arising from prolonged technology use include excessive technology usage. The second dilemma is thus referred as the 'excessive utilisation type', whereby the user regards the enhanced ability as a natural ability enhancement while largely disregarding the technology.

The next section proposes design approaches for resolving the two types of dilemmas.

\section{APPROACHES TO ADDRESSING DILEMMA}

\subsection{Use of continuous technology for resolving the decreased ability-type dilemma}

The first approach aims to overcome the loss of natural ability when a technology is discontinued. The aim is to ensure that all users can continue to operate the technology.

For example, the skill of starting a fire with wood is almost certainly redundant when cooking in modern urban environments. Most modern cooking technologies only require users to turn the knob on an electric or gas oven. The heating mechanism is already embedded in the gas oven, or in the electrical control of an induction heating cooker; manual ignition is no longer required.

In this situation, the decreased ability to light fires for cooking is circumvented by technology that is available at anytime and anywhere. That is, widespread deployment of cooking technologies will remove any problems incurred by loss of fundamental cooking skills.

Unlike air and gravity, technologies are not universally available, so design approaches that mitigate problems must consider the availability degree of the technology use. In the ideal case of highest availability, the technology will be ubiquitously present in the environment. In the case of lowest availability, the technology cannot function at all. Figure 1 shows the degrees of availability and the positions of several approaches on the availability continuum.

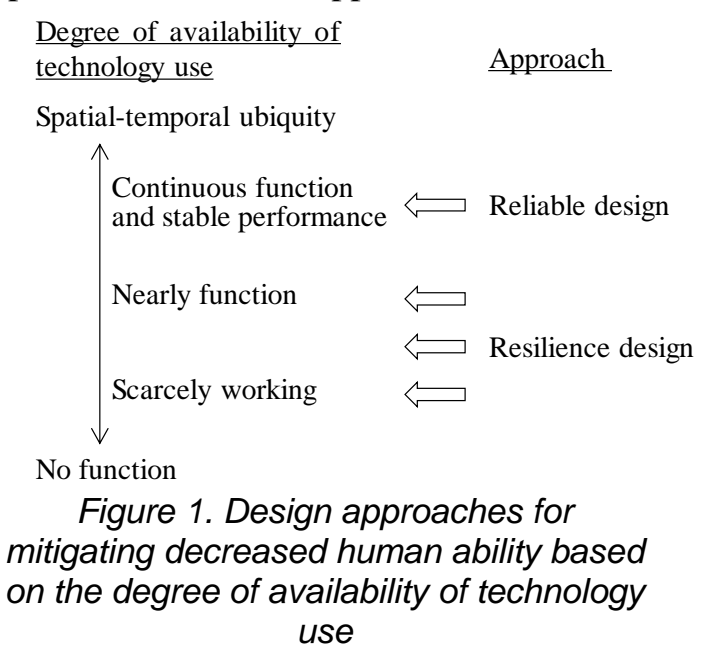

Reliable design is a typical approach that ensures the continuous function and stable performance of a system (Birolini, 1999, etc.). Infrastructures based on reliable design include water, electricity and transportation. These utilities are constructed redundantly to maintain high system functioning when problems occur. Constant maintenance also mitigates the problems.

Next, when the systems are required to function from perfunctory to nearly excellent, a resilience design approach is applied (Woods, 2006, etc.). Especially in Japan, this approach has attracted attention after large natural disasters. Resilience design is necessary for large infrastructure systems that widely impact on social activities after failure. 
It also targets medical systems and air-traffic control systems requiring absolute safety to prevent loss of life. Resilience design has shifted its approach from removing the cause of a problem for safety reasons (Safety-I) to flexibly responding to changing situations (Safety-II) (Hollnagel, 2014).

The above-mentioned approaches are generally known as spread design methods. Problems arising from the human use of technologies have not been tackled by this approach.

\subsection{Mitigating the decreased ability-type dilemma}

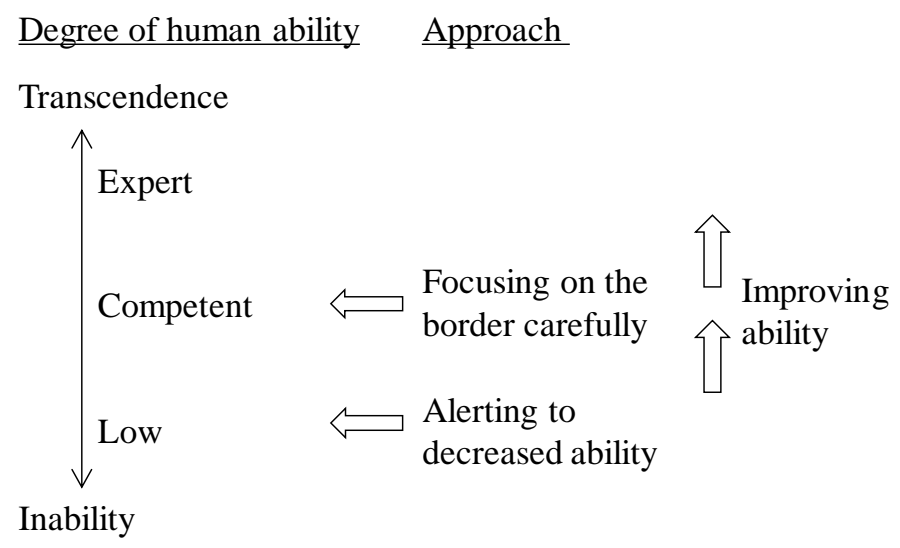

Figure 2. Design approaches for technologies compatible with degrees of human ability
The second approach is arresting the loss of ability when a technology is removed, which involves improving the human ability. In this approach, the technology is designed to match the degree of human ability and the degree to which the technology substitutes human functions. In essence, this approach focuses on human ability itself rather than the integration of human and technological functionalities.

Given that most humans exhibit moderate ability for a given task, technology advances human ability in a positive and transcendental direction. However, technology also reduces the natural ability of humans. The design approaches matched to the various degrees of human ability are shown in Figure 2.

\subsubsection{Improving human ability}

The human-ability dilemma can be mitigated by designing technologies toward improving human ability. Specifically, low ability can be advanced to competency, competency to high ability, and high ability to expert ability. Targeted training and educational methods can improve physical ability in sports and cognitive ability in intellectual pursuits.

For example, in sports training, the players' bodily motions are measured by a motion capture system with sensors, and the analysed data reveal the defects needing improvement. These sensor-base systems are used only during training, and are disallowed in real competitions. This approach aims to maintain and improve human ability when the technology is removed or invalidated.

The author's team has focused on the continued improvement of human skills. Athletes may reach a performance plateau, overcoming which requires various ideas. According to the author's team, the ability to overcome the stable plateau is requisite for improvement. The team has thus focused on methodologies involving physical and occupational therapies, which improve the players' motor ability. Through methods based on the modification of neurocognitive rehabilitation, the team has designed an approach that allows users to reorganise their sensor and motor abilities. In this approach, users discern the subtle changes in their joint positions and muscle tension, create the image of ideal motions, and explores the boundary between ability and inability. In particular, the tools developed by the team increase the degrees of freedom of the upper-limb motions by a robotic system (Koike, 2014, etc.), and improve cyclists' pedalling skills by adjusting the pedal load to suit the crank angle (Yubuki, 2018, etc.).

\subsubsection{Focus on the ability-disability border}

Most of the published approaches mitigate the decreased-ability dilemma by improving the overall human ability (see 4.2.1). However, the author's team considers that a variety of approaches is necessary to address the dilemmas. If technology use might reduce a moderately competent human to a less competent human, the ability reduction should be considered and arrested by an analytical approach. 
For example, wearable robots have been deployed at real worksites. Such robots can assist humans in lifting heavy objects that they cannot lift without tools. In tasks requiring more power than humans can exert, the loss of human ability by utilising the technology is inconsequential. However, when a human can accomplish a task sufficiently without a tool, the degradation of muscle activity after longterm use of the technology rouses concern. To address this problem, we must investigate to what extent technology usage causes muscular weakness, and include cautionary measures and suggestions in the technology design.

The author's team has proposed assistive technology that continues rather than replaces human activity. The motor behaviour of this technology removes only the highest load from the human. One such device assists the holding and lifting down of heavy materials. The Ministry of Health, Labour and Welfare of Japan recognises the back damage caused by continuously moving heavy loads (over $20 \mathrm{~kg}$ ) as a work-related accident. The author's team has devised a device with a brake mechanism that reduces the highest load of up to $20 \mathrm{~kg}$ to within the safety level of the workers (Hatano, 2018). Another tool assists users in descending a mountain. As the number of middle-aged and elderly persons climbing mountains increases, the number of mountain accidents caused by them increases. Moreover, as descent imposes a larger load on the leg muscles than ascending, muscle fatigue leading to injury is more likely while descending. The brake mechanism of our tool only removes the highest load on the thighs while climbing down, thereby alleviating fatigue and avoiding injuries (Esaka, 2017).

The technologies designed by this approach are intended for humans with moderate ability, necessitating careful consideration of the ability-inability boundary. Based on the load strength and working hours of users, the technology can be designed to ensure the safety and security of workers without over-assisting them.

\subsubsection{Focus on decreased ability}

The above approaches focus on the ability situation of users. In reality, few cases of technology usage definitively decrease the ability of users. When technologies do cause such problems, they are redesigned or removed, so are unavailable in the real world. However, the cyborgs, brain-machine interfaces and brain-computer interfaces currently being developed might exert stronger effects on mind and body than existing technologies, raising the alarm level of the decreased-ability dilemma. Even if the decreased ability locates within the allowable range during experimental trials, out-ofrange cases must be assumed in the wider community. Preparations for such cases are imperative; at least, they would alert stakeholders to the possible limitations of the technology. The mitigation suggestions of stakeholders will provide valuable feedback for the technology design.

Another approach allows a temporary reduction of users' ability to highlight the problems. Examples are simulation suits for aged persons and hemiplegia patients. The sensory abilities of users are temporarily reduced by wearing coloured glasses, narrow-view goggles and earplugs. Meanwhile, motor ability is reduced by attaching weights and restraints on limbs.

To generate a realistic experience of hemiplegia in able-bodied users, the author's team has focused on the recorded comments of hemiplegia patients, such as 'I don't know how to move my leg and arm', and 'My leg moves automatically against my will'. The author's team interpreted such experiences as the variance between the sensed and physical bodily actions. The team then devised a tool that generates illusory kinaesthesia and reflex actions, allowing users to experience such variance (Wesugi, 2013).

These technologies will be available for educating not only young medical-service workers, but also designers of living environments and the families of patients afflicted with hemiplegia and similar conditions.

Moreover, potential stakeholders using these technologies will be able to imagine the decreased ability of patients with physical impairments.

\subsection{Regulating the excessive utilisation of technology}

The excessive-utilisation dilemma can be resolved by two main approaches. The first approach regulates the technology use to within moderate levels. The direction of this approach opposes that suggested in 4.1, which mitigates the decreased ability by continuing the technology use as far and widely as possible. For example, users cannot easily regulate the excessive use of their smartphones. This problem is popularly solved by the pay-as-you-go method, which converts the amount of utility time to a monetary amount. The utility time of users is then regulated by the cost restriction. Among the approaches that manage the usage conditions and establish moderate habits are Persuasive Design 
(Fogg, 2002) and Nudge (Thaler, 2009). These approaches promote lifestyle improvements such as regular exercise habits and cessation of smoking. Users are motivated and triggered to achieve the design goals by quantifying user activity and competing with other users in a game environment.

Persons other than the users can

Degree of regulating
technology use

Strong

$\uparrow$

$\longleftrightarrow$ Strict restriction

$\Leftarrow$ Interventional restriction by other beings

Promotional self-regulation

Self-regulation

Weak

Figure 3. Design approaches for regulating the use of technologies regulate technology usage; for instance, parents can manage their children's smartphone use. Much more strictly, technology use can be managed by an administrator issued with a specific licence; for example, a medical doctor alone can prescribe medicines to a patient.

Figure 3 matches the above-mentioned solutions to their degrees of regulating technology use. In order of weak to strong regulation, these solutions are self-regulation, promotional selfregulation, interventional restriction by persons and artificial agents other than users, and strict restriction by an administrator.

\subsection{Reducing the negative social effects of excessive technology use}

The second approach for solving the excessive-utilisation dilemma tackles human disposition, which changes after excessive technology usage (as described in 4.2). When resolving the decreased-ability dilemma, the reference is the human ability in the absence of the technology. However, when exploring the change in human disposition, the disposition should be assessed in the absence and presence of the technology. Figure 4 shows the approaches for improving the disposition of technology users.

Given that most users have a moderate disposition, we can assume improved and worsened situations. In the positive direction, technology use can promote a person's disposition from worse to moderate or from moderate to better. For example, wearing a favourite wristwatch or clothing can improve one's confidence or happiness level. The basic functions of watches and clothes are timekeeping and regulating body temperature, respectively, but the designs of both items can improve the human disposition.

Improving human disposition causes no problems at least, and promotes mental health at best. In particular, users actively integrate with their favoured designs, enhancing their happiness and wellbeing (Calvo, 2014) (Jimenez, 2015). However, the negative effects of technology on human disposition, and the amount of technology usage that exerts this effect, need to be investigated. Therefore, precautions and suggestions against lowered disposition, as well as decreased ability,

Degree of alteration in human disposition

Approach

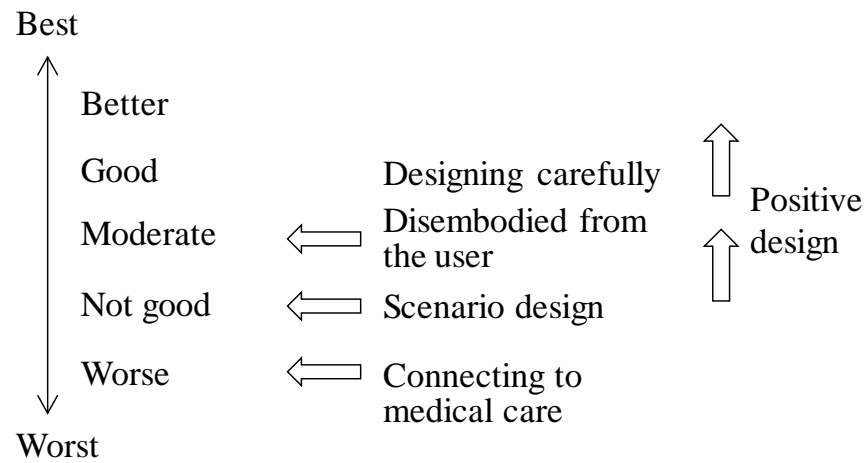

Figure 4. Design approaches for improving the disposition of technology users should be included in the technology design. In this approach, users should be aware of their technology usage; that is, the technology should be disembodied from the user.

In real life, this negative effect might be unrecognised beforehand, and will be immaterial if the benefits of the technology outweigh the disadvantages. Therefore, the approach should prepare for the assumed worst situation. However, whereas letting users temporarily experience a reduction in ability is useful in some situations (see 4.2), a technology that allows users to experience a poor human disposition is 
ethically reprehensible. Therefore, an effective approach would create scenarios of negative dispositions that attract the attention of stakeholders, encouraging them to discuss the problems raised in the scenarios. In the worst cases, such as internet addiction, the approach must be connected to medical cares.

\section{DISCUSSION}

Until recently, various dilemmas related to technology use have remained unsolved and neglected. To promote solutions to these dilemmas, the already spreading existing approaches should be further advanced. This section examines the possibility of advancing the most-applied approach described in 4.1. As an example, we consider the evolution of a ubiquitously available technology, the mobile phone. Towards mobile-phone coverage at any time and place, base-station antennas have been deployed in many places, even in underground malls and mountaintops. Meanwhile, the usage time of mobile phones has been extended by installing larger electrical capacitances. Generically, the technology has evolved towards continuous utilisation by many users, driving the further development of the technology.

Can further promotion of this movement resolve the decreased-ability dilemma?

Ultimately, this technology might become as ubiquitous as air and gravity. In modern city life, technology for igniting a fire for cooking and heating has become so widespread that manual firelighting is no longer required (as described in 4.1). However, as the loss of this skill is completely hidden, the decreased-ability dilemma is fundamentally unsolved.

Recently, Japan has been damaged by several large-scale natural disasters that widely disrupted the gas, electricity and oil supplies around the disaster-stricken areas. Familiar technologies were suddenly unavailable and the inhabitants of damaged areas depended heavily on external support. These situations remind us that even fundamental technologies such as fire-making might be required at some time and place. Anti-disaster measures suggest that gas cookers and stoves independent from the existing infrastructure should be dispersedly stored in each household, or as common utilities in apartment complexes. Besides improving the reliability and resilience of single large infrastructures, these approaches widely disseminate local and independent technologies. Fundamental technologies such as fire-lighting and obtaining drinkable water are considered redundant in modern city life. However, a variety of fundamental technologies would reduce the great social confusion after a large system is disrupted by a natural disaster.

Such fundamental technologies are beneficial not only in emergency situations, but also in regions where drinkable water and fuel are not readily obtainable. Recently, such social problems in developing regions have been solved by designing the utilisation of local resources (Smith, 2007). For example, Philips Design has designed a wood stove with high combustion efficiency and low maintenance requirements for poor households in India. Since their introduction, the stoves have been spreading via non-profit activity (Chulha, 2007). The discussed approaches are relevant not only to water and fuel infrastructure technologies, but also to necessary living items such as eye glasses, whose power can be adjusted by the amount of silicone oil instead of optical lens. The fundamental technologies are usually produced for people who use them in daily life, and are imported to developed areas facing emergency situations such as large natural disasters. Designing fundamental technologies both reduces the decreased-ability dilemma and assists developing areas. The discussed approaches will contribute to a sustainable society as well as benefitting individual users.

\section{CONCLUSION}

Technology can benefit users by extending their range of abilities, but can also decrease their natural abilities or (when used excessively) their dispositions. The decreased-ability and excessive-use dilemmas are usually identified and tackled after the social consequences have become severe. Due to the multistability of technologies, all possibilities of technology use cannot be anticipated beforehand. However, the predictable dilemmas can be considered in the design process. In this paper, the author suggests several approaches for mitigating these dilemmas.

To examine the dilemmas in technology usage, the author first referred to Shibata's analysis of McLuhan's extension theory and revealed the two dilemmas of decreased human ability and excessive usage. The decreased-ability dilemma can be mitigated by continuous technology use and by designing technology compatible with the degree of human ability. Meanwhile, the excessive- 
utilisation dilemma can be resolved by regulating the technology use and designing technology that elicits the desired change in human disposition. Next, the possibility of advancing the existing approaches was discussed. It was suggested that fundamental technologies can address both the individual dilemma of decreased human ability and the wider problems in developing areas.

In future work, the author will investigate other dilemmas not raised in the early literature, and address them by extending the proposed approaches. Moreover, the author will apply these approaches to the design of specific technologies.

Importantly, technology designers should assume that non-negligible dilemmas can occur whenever their technologies co-exist with humans. From this viewpoint, various stakeholders can understand the negative and positive implications of continuous technology use.

\section{REFERENCES}

Birolini, A. (1999), Reliability Engineering Theory and Practice, Springer.

Calvo, R.A. and Peters, D. (2014), Positive Computing: Technology for Wellbeing and Human Potential, MIT Press.

Chulha, https://www.chulha.org/

Esaka, N., Shinkawa, K. and Wesugi, S. (2015), “Assistance for Moving with Eccentric Contraction Development of Braking Mechanism for Knee Flexion in Stance Phase on Descending a Mountain", Proc. of the Human Interface Symposium 2015(HIS), pp. 427-430

Fogg, B.J. (2002), Persuasive Technology: Using Computers to Change What We Think and Do, Interactive Technologies, Morgan Kaufmann.

Hollnagel, E. (2014), Safety-I and Safety-II: The Past and Future of Safety Management, CRC Press.

Hatano, Y., Kawano, A., Kobayashi, S., Fujita, T., Tomita, R. and Wesugi, S. (2018), "Supporting Method for Carrying Heavy Loads Focused on Construction Sites (Development of a Tool to Assist Holding and Lifting Down Long Objects with Braking Mechanism)", Proc. of the Symp. on sports and human dynamics 2018(JSME), Vol. 2018, p. D-24

Ihde, D. (1990), Technology and the Lifeworld: From Garden to Earth, Indiana University Press.

Jimenez, S., Pohlmeyer, A. and Desmet, P. (2015), Positive Design Reference Guide, blurb.

Koike, M., Omurao, T. and Wesugi, S. (2014), "Design of a Supporting Method for Finding Clues to Construct Unfamiliar Motions - Development of an Interface System Focusing on the Skeletal Structure and the Haptic Characteristics for the Scapula Motions", Human Communication Group Symposium 2014, pp. 497-501

Plato, Phaedrus, http://classics.mit.edu/Plato/phaedrus.html

Shibata, T. (2013), McLuhan to media-ron -shintairon no syugou, keiso shobo

Shibata, T. (2015), "The "archetype" of cyborg: Understanding J. D. Bernal on genealogy of "extension"”, Annual Bulletin of the New Humanities, Vol. 12, pp. 42-91.

Smith, C.E. (2007), Design For The Other 90\%, Cooper Hewitt, Smithsonian Design Museum.

Thaler, R.H. and Sunstein, C.R. (2009), Nudge: Improving Decisions About Health, Wealth and Happiness, Penguin.

Tsuchiya, K. (2001), Kangaekata wo kangaeru: souzousei wo hagukumu hinto, Ohmsha Ltd.

Verbeek, P.P.C.C. (2011), Moralizing Technology: Understanding and Designing the Morality of Things, University of Chicago Press.

Verbeek, P.P.C.C. (2015), "Cover story: Beyond Interaction: a short introduction to mediation theory”, Interactions (ACM), Vol. 22 No. 3, pp. 26-31. http://doi.org/10.1145/2751314.

Wesugi, S. (2013), "Design Approach of Simulation Exercise with Use of Device and Its Significance: Design of Novel Device for Realistic Experience of Being a Hemiplegia Patient", Int. Conf. on Human-Computer Interaction, Springer, Lecture Notes in Computer Science 8017, pp. 315-324

Woods, D.D. and Hollnagel, E. (2006), Resilience Engineering: Concepts and Precepts, CRC Press.

Yubuki, S., Tokuyasu, Y., Iwata, S., Kawakami, Y. and Wesugi, S. (2018), "Improvement Method on Pedaling Skills of a Road Bike (Development of Training Equipment with Local Braking Applied to Pull-up Action)", Proc. of the Symp. on sports and human dynamics 2018(JSME), Vol. 2018, p. B-23

Zhuang-zi, https://ctext.org/zhuangzi

\section{ACKNOWLEDGEMENTS}

This paper forms part of the research performed under a Waseda University Special Research Period. The author would like to thank Prof. Peter-Paul Verbeek for his support during my stay at the University of Twente. The author would also like to thank Enago (www.enago.jp) for the English language review. 